\title{
Efficacy and Safety of OnabotulinumtoxinA in Patients With Neurogenic Detrusor Overactivity Caused by Spinal Cord Injury: A Systematic Review and Meta-Analysis
}

\author{
Guang-Ping Li*, Xiao-Yan Wang*, Yong Zhang \\ Department of Urology, Beijing Tian Tan Hospital, Capital Medical University, Beijing, China
}

Purpose: OnabotulinumtoxinA (BoNT-A) is a promising therapy for treating neurogenic detrusor overactivity (NDO) in individuals with spinal cord injury (SCI). This systematic review and meta-analysis aimed to carry out an in-depth review and to make an objective estimation of the efficacy and safety of BoNT-A on NDO after SCI.

Methods: The PubMed, Embase, and Cochrane databases were searched for all relevant articles published from 2001 to 2016 that referred to NDO, SCI, and BoNT-A or botulinum toxin A. All data were recorded in an Excel spreadsheet by 2 individual reviewers. Review Manager version 5.3 was used to carry out the meta-analysis.

Results: This analysis included 17 studies involving 1,455 patients. Compared with placebo and baseline, BoNT-A was effective in increasing maximum cystometric capacity, volume at first involuntary detrusor contraction, cystometric bladder capacity (all $\mathrm{P}<0.00001)$, compliance $(\mathrm{P}=0.001)$, and the number of patients with complete dryness $(\mathrm{P}=0.0003)$, and decreasing detrusor pressure, the number of patients with no involuntary detrusor contractions, the maximum flow rate, the incidence of detrusor overactivity (all $\mathrm{P}<0.00001$ ), and the number of urinary incontinence episodes $(\mathrm{P}=0.001)$. There were no statistically significant differences between doses of $200 \mathrm{U}$ and $300 \mathrm{U}$ or between injections into the detrusor and submucosa. There were no life-threatening adverse events.

Conclusions: BoNT-A is effective and safe in treating NDO after SCI. There were no statistically significant differences between doses of $200 \mathrm{U}$ and $300 \mathrm{U}$ or between injecting into the detrusor and submucosa. However, more high-quality randomized controlled trials are still needed.

Keywords: OnabotulinumtoxinA; Meta-analysis; Neurogenic detrusor overactivity; Spinal cord injury

- Fund/Grant Support: This research was supported by Beijing Municipal Administration of Hospitals Clinical Medicine Development of Special Funding Support, code: ZYLX201820.

- Conflict of Interest: No potential conflict of interest relevant to this article was reported.

\section{INTRODUCTION}

Spinal cord injury (SCI) is a devastating event that can cause far-reaching effects on individuals' health, with further economic and social ramifications [1]. The incidence rate of SCI hospitalizations was found to be 4.8 per 1,000 person-years [2].

In patients with severe SCI, the micturition reflex from the spinal cord segment is released from supraspinal modulation. This reflex increases the concentration of nerve growth factor distributed in the bladder wall. Subsequently, hyperexcitability
Corresponding author: Yong Zhang (iD https://orcid.org/0000-0001-6534-8217 Department of Urology, Beijing Tian Tan Hospital, Capital Medical University, No 6 Tiantan Xi Li, Dong Cheng District, Beijing 100050, China

E-mail: 18810665538@163.com / Tel: +86-10-6709-8393 / Fax: +86-10-6709-6611 ${ }^{*}$ Guang-Ping Li and Xiao-Yan Wang contributed equally to this study as co-first authors. Submitted: May 27, 2018 / Accepted after revision: August 25, 2018 
of afferent nerves and detrusor overactivity (DO) occur in the bladder [3]. Then, patients often complain of lower urinary tract symptoms such as urinary frequency, urgency, and urinary incontinence (UI) [4]. Therapies for neurogenic detrusor overactivity (NDO), which is a complex and multilevel process, include medications (such as anticholinergic drugs and onabotulinumtoxinA [BoNT-A]), surgical procedures (such as sacral nerve stimulation), functional exercises, and psychological therapies. Since the causes of NDO are diverse, therapies should be chosen on an individualized basis $[5,6]$.

In August 2011, BoNT-A was approved for patients with NDO after SCI by the U.S. Food and Drug Administration (FDA) [7]. To date, many studies have focused on the efficacy and safety of BoNT-A in improving urodynamic parameters and reducing the number of UI episodes. However, the absence of high-quality studies, especially randomized controlled trials (RCTs), has made it challenging to conduct a systematic review of this issue. Therefore, the efficacy and safety of BoNT-A have remained controversial. With this in mind, the purpose of this meta-analysis was to further evaluate the efficacy and safety of BoNT-A.

\section{MATERIALS AND METHODS}

\section{Search Strategy}

We searched the PubMed, Embase, and Cochrane databases for all relevant articles published from 2001 to June 2016.The references of the retrieved studies were also reviewed. We used the following keywords: "neurogenic detrusor overactivity," "spinal cord injury," "botulinum toxin A," "BoNT-A" and "BOTOX." Articles were restricted to clinical trials.

\section{Inclusion Criteria}

All identified clinical trials were screened by 2 reviewers and had to meet the following criteria for inclusion in our analysis: (1) The participants were older than 18 years. (2) NDO was mainly caused by SCI (50\% or more of participants). (3) BoNTA was administered by injection into the bladder wall. (4) The study contained accessible data on maximum cystometric capacity (MCC), volume at first involuntary detrusor contraction (VFIDC), detrusor pressure (Pdet), UI episodes, compliance, voiding volume, postvoid residual volume (PVR), maximum flow rate (Qmax), mean functional bladder capacity, the number of patients with complete dryness (CD), incidence of DO, incontinence-specific quality of life (I-QoL), and the number of patients with no involuntary detrusor contraction (IDC). (5) The full text of the study could be found. Studies not meeting any of those criteria were excluded.

\section{Exclusion Criteria}

Studies meeting any of the following criteria were excluded from this analysis: (1) The full text was not accessible. (2) It was not a clinical trial (i.e., reviews, basic science). (3) It contained duplicate data from the same trial; in such cases, where findings from the same trial were published in multiple journals, only the most useful publication was used for this analysis. (4) Studies without sufficient subjects (i.e., case reports of single cases) or accessible data were also excluded from this analysis. After the selection, we regathered all the articles, both included and excluded, to perform a confirmatory assessment of the reasons for inclusion or exclusion.

\section{Quality Assessment}

All the studies were graded according to the Cochrane Handbook for Systematic Reviews of Interventions 5.1. Eight entries represented 5 biases: selection bias, performance bias, detection bias, attrition bias, and reporting bias. Random sequence generation, double-blinding of participants and studiers, and double-blinding of outcomes indicated low risk. Allocation concealment, incomplete outcome data addressed, and selective reporting corresponded to high risk. Based on these criteria, all studies were classified using 3 grades, from A to C: (A) If all the key domains, and most information, met the low-risk criteria, the study was seen as having a low risk of bias. (B) If $\geq 1$ key domains had an unclear risk of bias, and most information was at low or unclear risk, the study was viewed as having an unclear risk of bias. (C) If $\geq 1$ key domains met the high-risk criteria and some information, with a significant influence on the results, was at a high risk of bias, the study was considered to have a high risk of bias [8].

\section{Data Extraction}

Two reviewers read all the full texts, including the content of the publications and abstracts. The following information was collected from all included studies: (1) the first author's name and the year of publication; (2) the nations of the participants; (3) the sample size and numbers of each group; (4) the dose and location of administration; (5) baseline, index after at least 1 injection, or changes in urodynamic parameters and clinical outcomes; and (6) the type and number of adverse events (AEs). If 
there was controversy over any aspects of the studies, a third reviewer was invited to review the study. If necessary, a fourth reviewer was included.

\section{Statistical Analysis}

All data were recorded in an Excel spreadsheet by 2 individual reviewers. After this procedure, Review Manager ver. 5.3 (Cochrane Collaboration, Oxford, UK) was used to carry out the meta-analysis. In this process, the mean difference (MD) and 95\% confidence interval (CI) were calculated using the software. Meanwhile, $\mathrm{I}^{2}$ was used to measure heterogeneity across trials. If $\mathrm{I}^{2}$ did not reach $50 \%$, we chose a fixed-effects model. When $\mathrm{I}^{2}$ exceeded 50\%, a random-effects model was more suitable. All types of AEs and the overall number of each AE in both the experimental and control groups were recorded.

\section{RESULTS}

\section{Characteristics of Individual Studies}

Fig. 1 shows the process of selection in this meta-analysis. After reading the abstracts and full texts, the 70 studies excluded involve (1) 64 excluded based on title and abstracts; (2) 2 excluded due to absent of full text; (3) 4 reviews, cases or editorials in Fig. 1. The remaining 24 studies involve 7 excluded (3 repetition and 4 no accessible data) and 17 eligible studies included. Ultimately, 17 studies with 1,455 patients were included in the meta-analysis. Eleven studies were RCTs. Eight studies investigated BoNT-A versus placebo, 4 investigated BoNT-A doses of 200 U versus 300

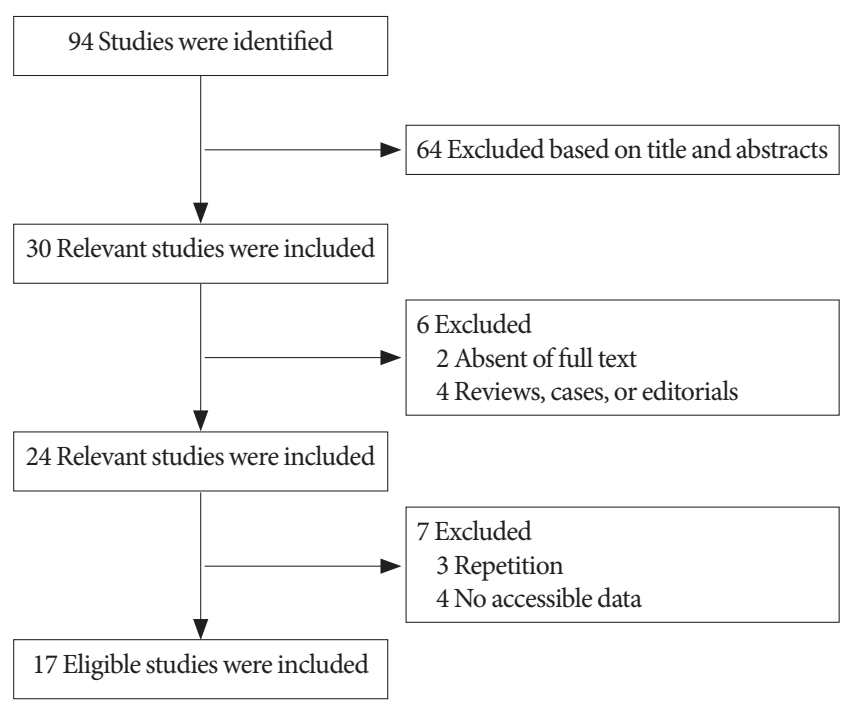

Fig. 1. The process of selection for this meta-analysis.
U, 2 investigated injections into the detrusor versus submucosa, and 2 investigated sparing the trigone versus avoiding the trigone. According to the Cochrane Handbook for Systematic Reviews of Interventions 5.1, 5 studies were evaluated as grade A, 5 as grade $\mathrm{B}$, and 7 as grade $\mathrm{C}$. The primary characteristics and quality assessment of the included studies are shown in Table 1 . The funnel plot provided an estimation of the publication bias of the studies, and no evidence of bias was found (Fig. 2).

\section{The Efficacy of BoNT BoNT-A versus placebo}

Five studies involving 961 participants included data for a comparison of BoNT-A versus placebo [9-14]. Compared with placebo, BoNT-A increased the MCC (MD, 134.75; 95\% CI, 105.06-164.44; $\mathrm{P}<0.00001)$, VFIDC (MD, 163.42; 95\% CI, 96.41-230.43; $\mathrm{P}<0.00001)$, and the number of participants with no IDC and decreased Pdet (MD, -30.48; 95\% CI, -38.38 to $-22.58 ; \mathrm{P}<0.00001)$ and $\mathrm{UI}$ episodes $(\mathrm{MD},-12.45 ; 95 \% \mathrm{CI}$, -19.90 to $-5.00 ; \mathrm{P}=0.001)$. However, the PVR (MD, 95.97; 95\% CI, 60.10-131.84; $\mathrm{P}<0.00001)$ increased more in the BoNT-A group than in the placebo group (Fig. 3).

\section{Baseline versus after treatment}

Ten studies involving 386 participants included data representing baseline versus posttreatment outcomes [10,15-24]. The results presented above were confirmed in the comparison between baseline and posttreatment values (Fig. 4) [9-21,24,25]. In addition, BoNT-A was effective in increasing the voiding volume (MD, 51.01; 95\% CI, 37.75-64.27; $\mathrm{P}<0.00001$ ), compliance $(\mathrm{MD},-14.34 ; 95 \% \mathrm{CI},-23.18$ to $-5.49 ; \mathrm{P}=0.001)$, and the number of patients with $\mathrm{CD}$ (odds ratio [OR], 0.02; 95\% CI,

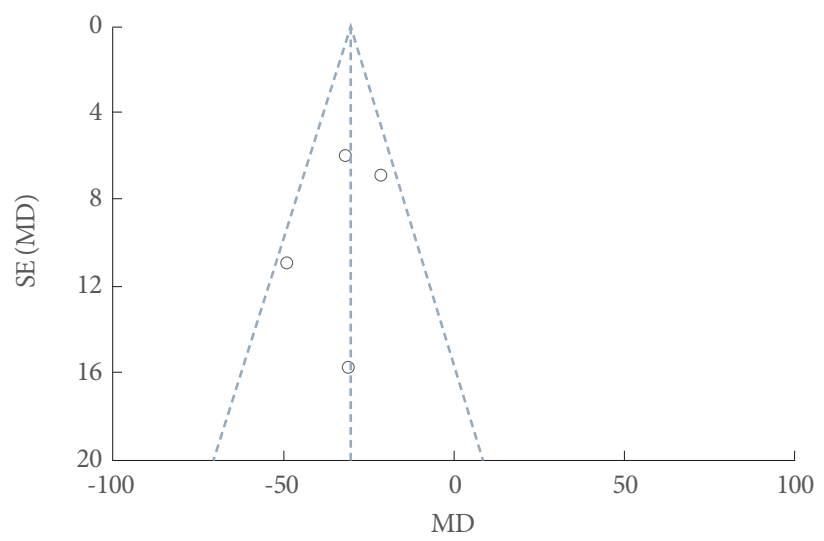

Fig. 2. Funnel plot of the studies included in the meta-analysis. $\mathrm{SE}$, standard error; $\mathrm{MD}$, mean difference. 


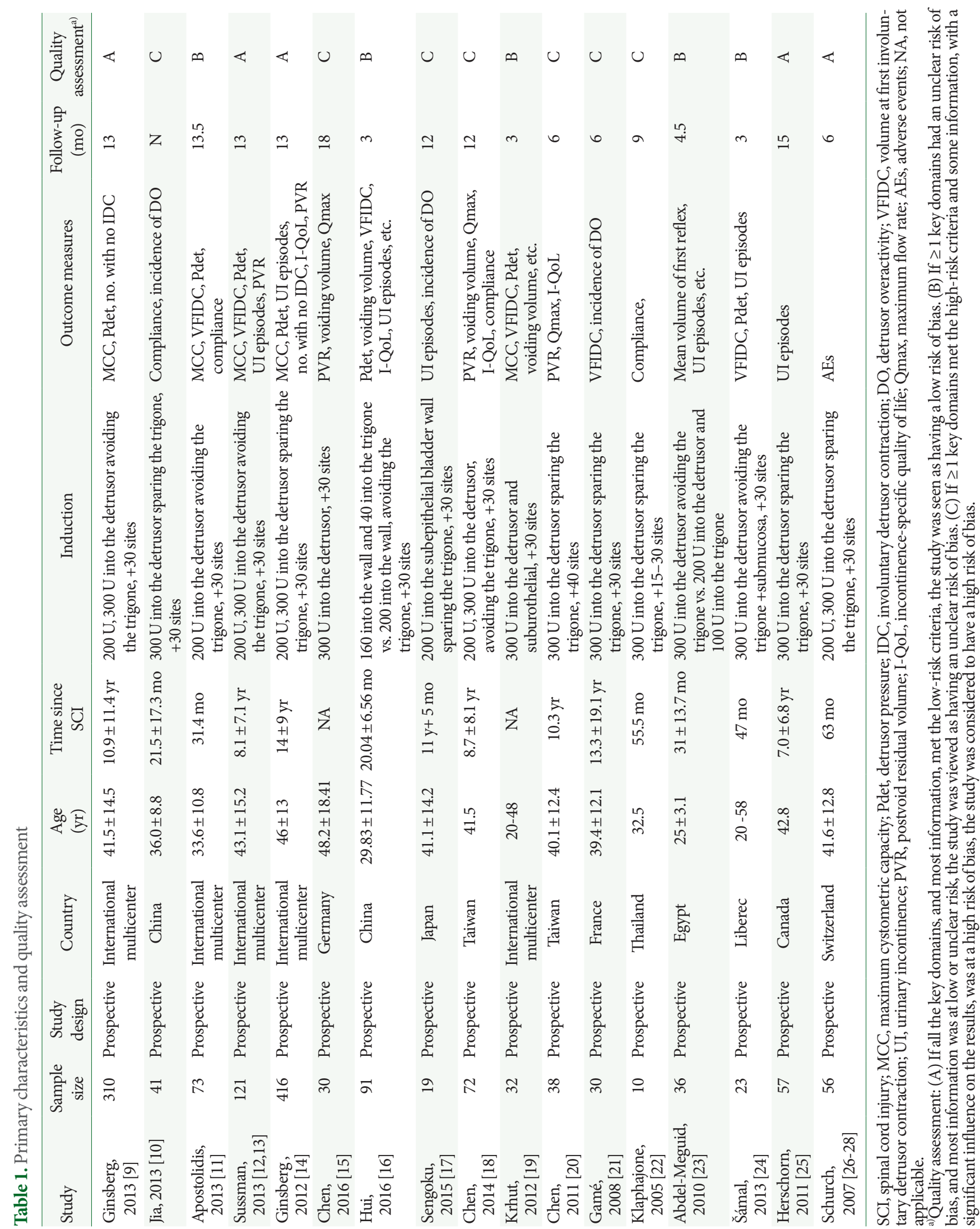




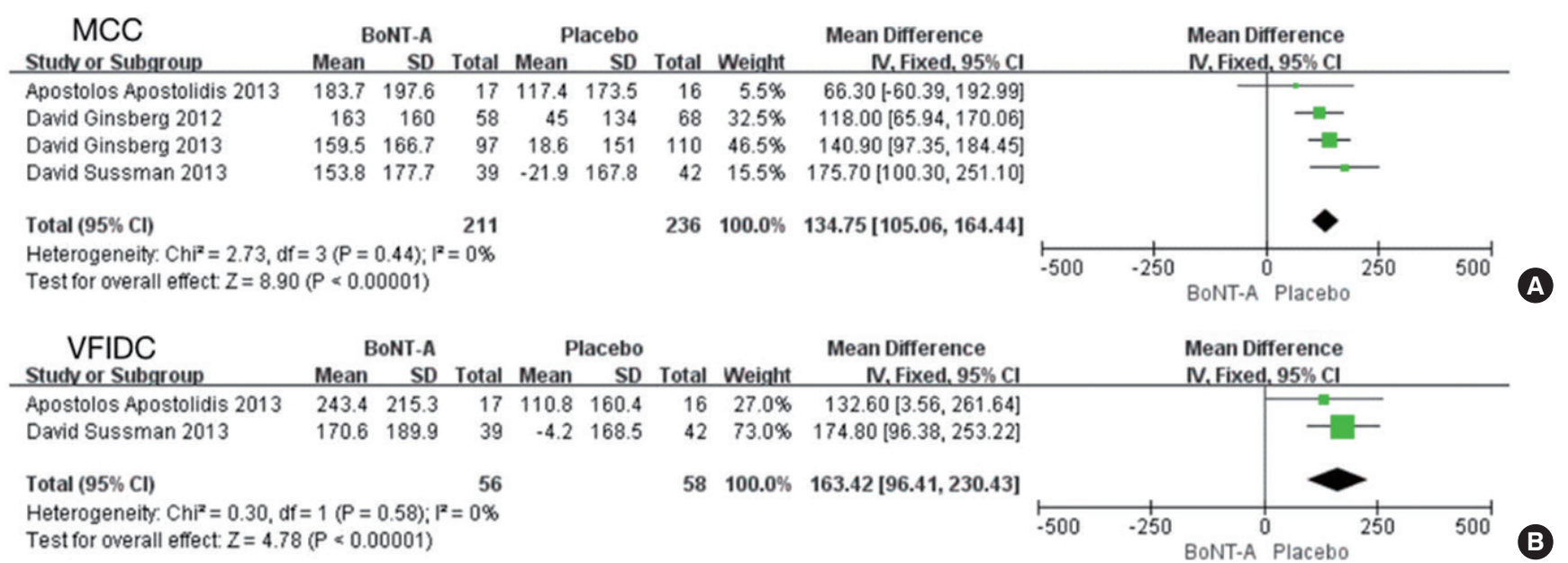

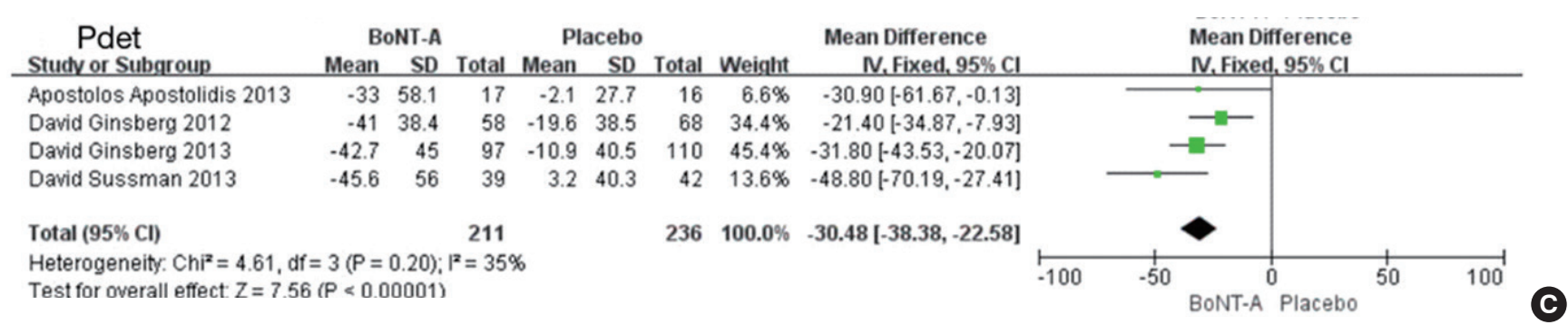

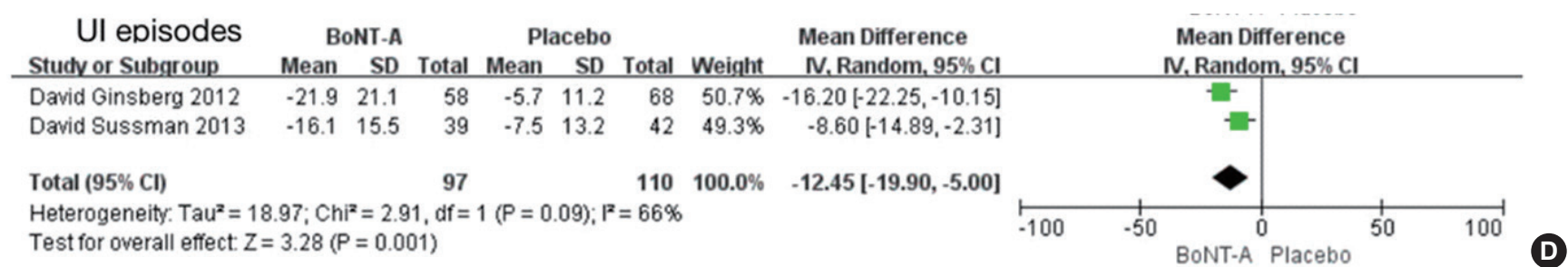

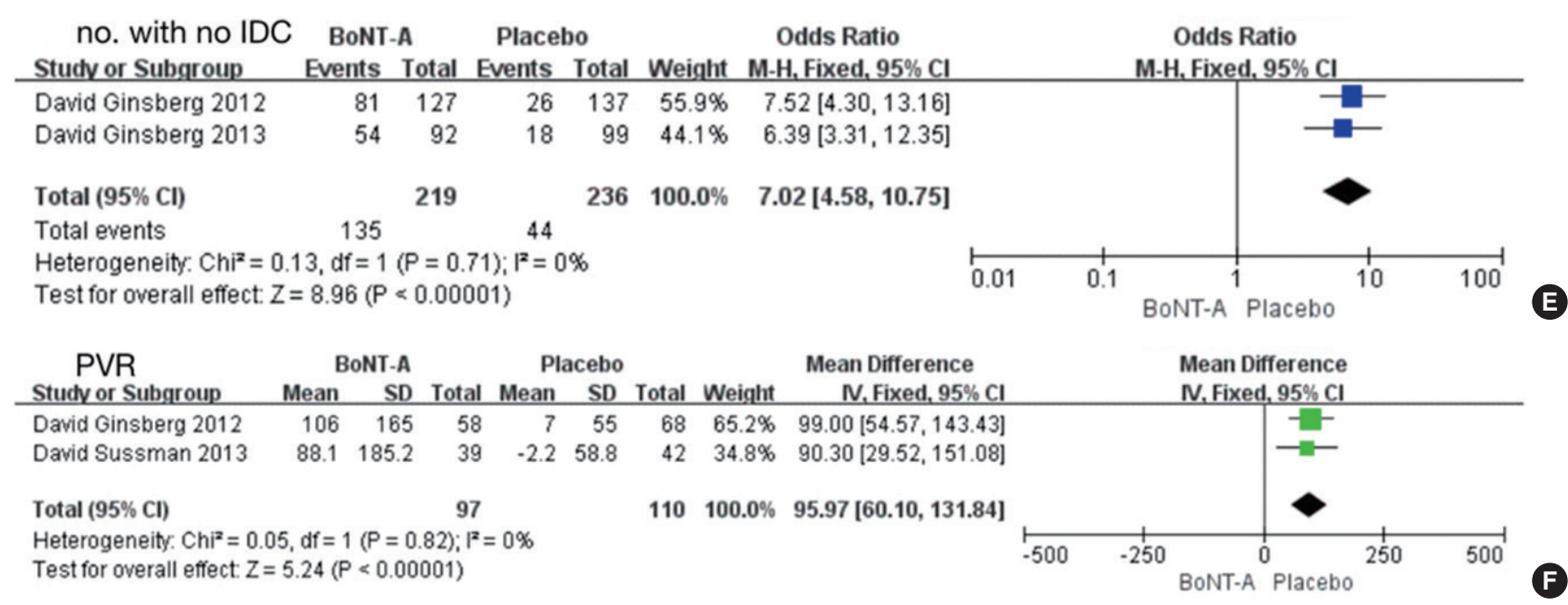

Fig. 3. Forest plots comparing the effects of onabotulinumtoxinA (BoNT-A) versus placebo on maximum cystometric capacity (MCC) (A), volume at first involuntary detrusor contraction (VFIDC) (B), detrusor pressure (Pdet) (C), urinary incontinence (UI) episodes (D), number of patients with no involuntary detrusor contraction (IDC) (E), and postvoid residual volume (PVR) (F). SD, standard deviation; $\mathrm{CI}$, confidence interval; df, degrees of freedom. 


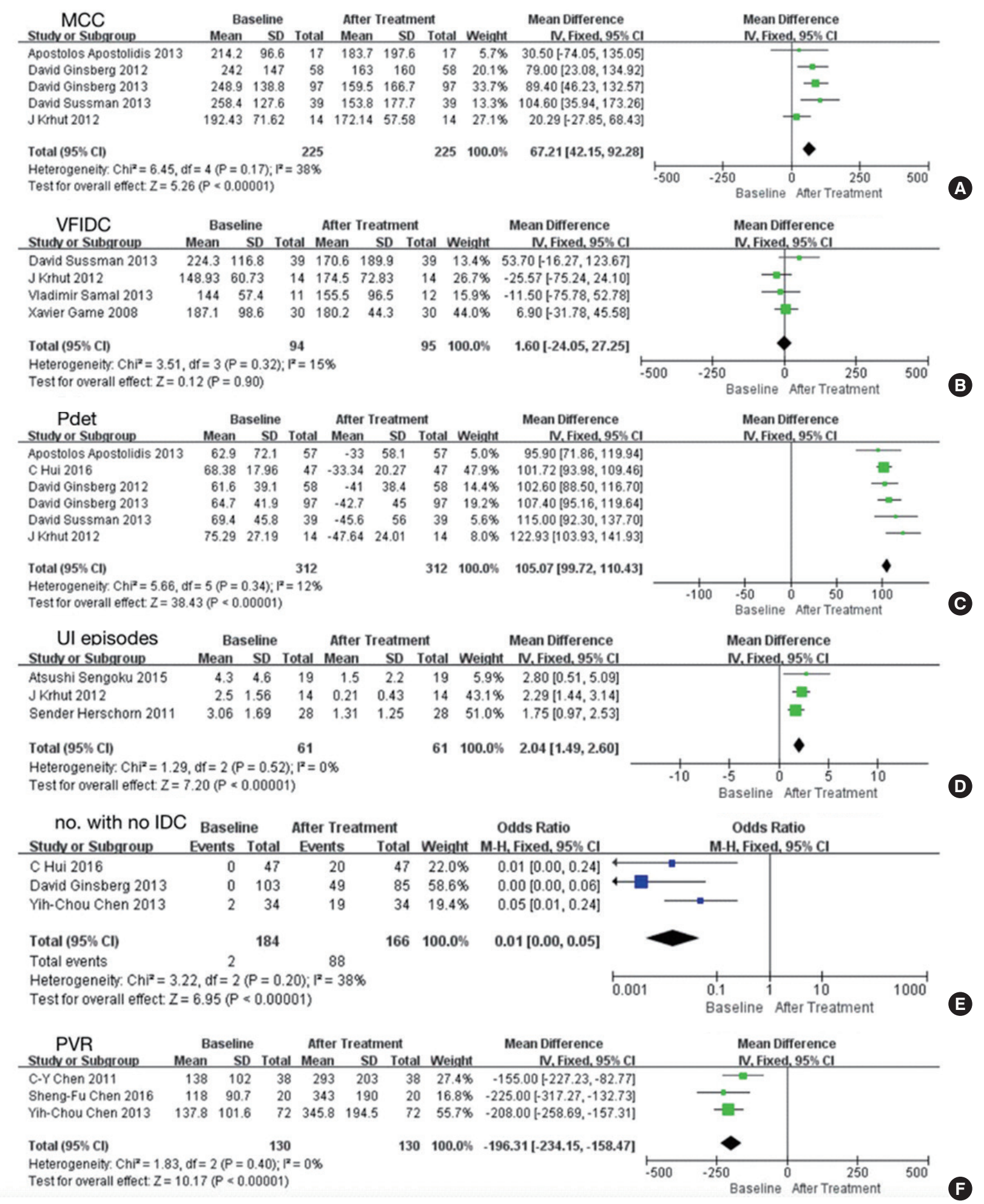

Fig. 4. Forest plots comparing the effect of onabotulinumtoxinA (BoNT-A) on baseline values versus posttreatment values of maximum cystometric capacity (MCC) (A), volume at first involuntary detrusor contraction (VFIDC) (B), detrusor pressure (Pdet) (C), urinary incontinence (UI) episodes (D), number of patients with no involuntary detrusor contraction (IDC) (E), and postvoid residual volume (PVR) (F). SD, standard deviation; CI, confidence interval; df, degrees of freedom. 


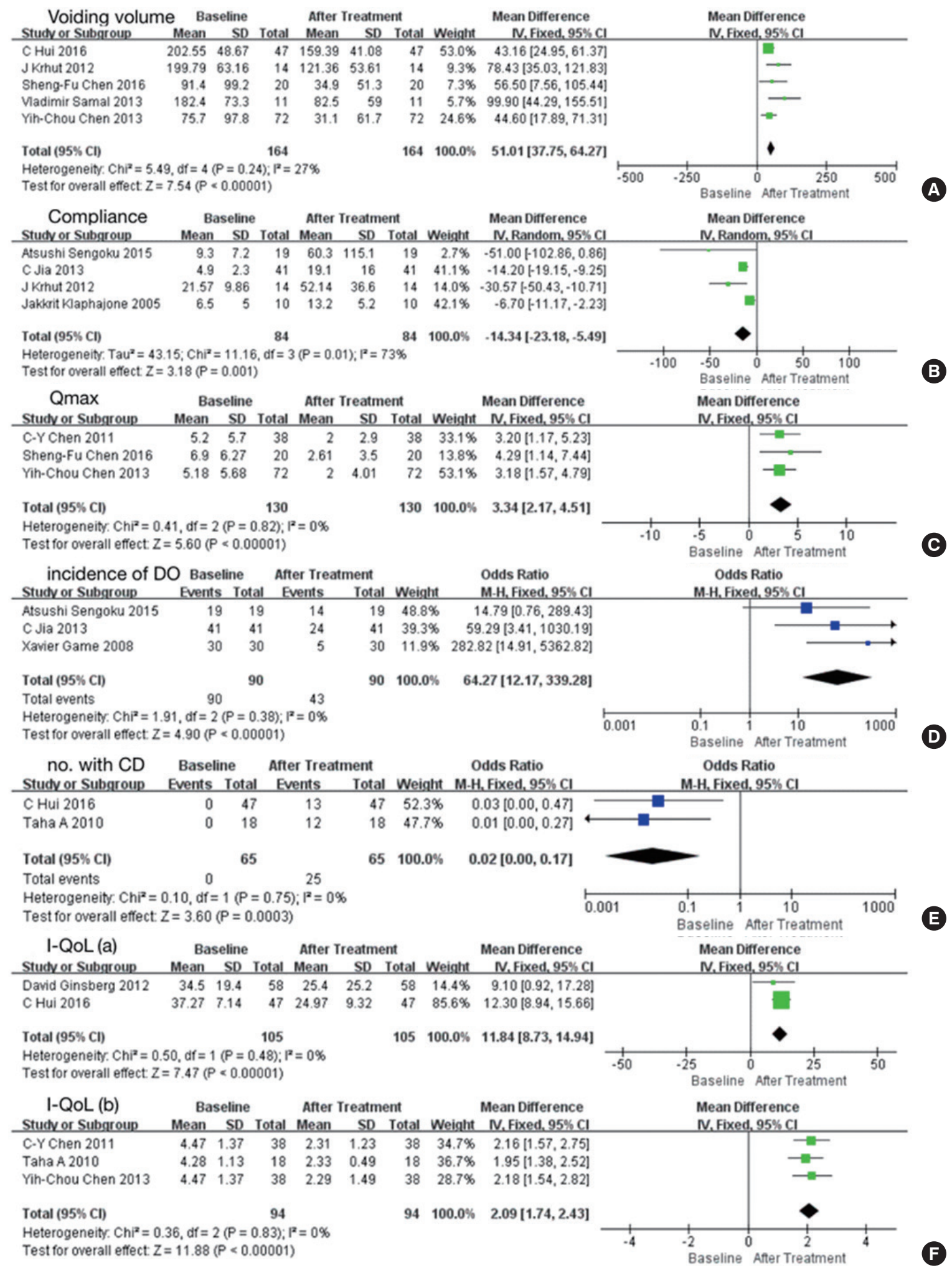

Fig. 5. Forest plots comparing the effect of onabotulinumtoxinA (BoNT-A) on baseline values versus posttreatment values of voiding volume (A), compliance (B), maximum flow rate (Qmax) (C), incidence of detrusor overactivity (DO) (D), number of patients with complete dryness $(\mathrm{CD})(\mathrm{E})$, and incontinence-specific quality of life (I-QoL) (F). SD, standard deviation; CI, confidence interval; df, degrees of freedom. 


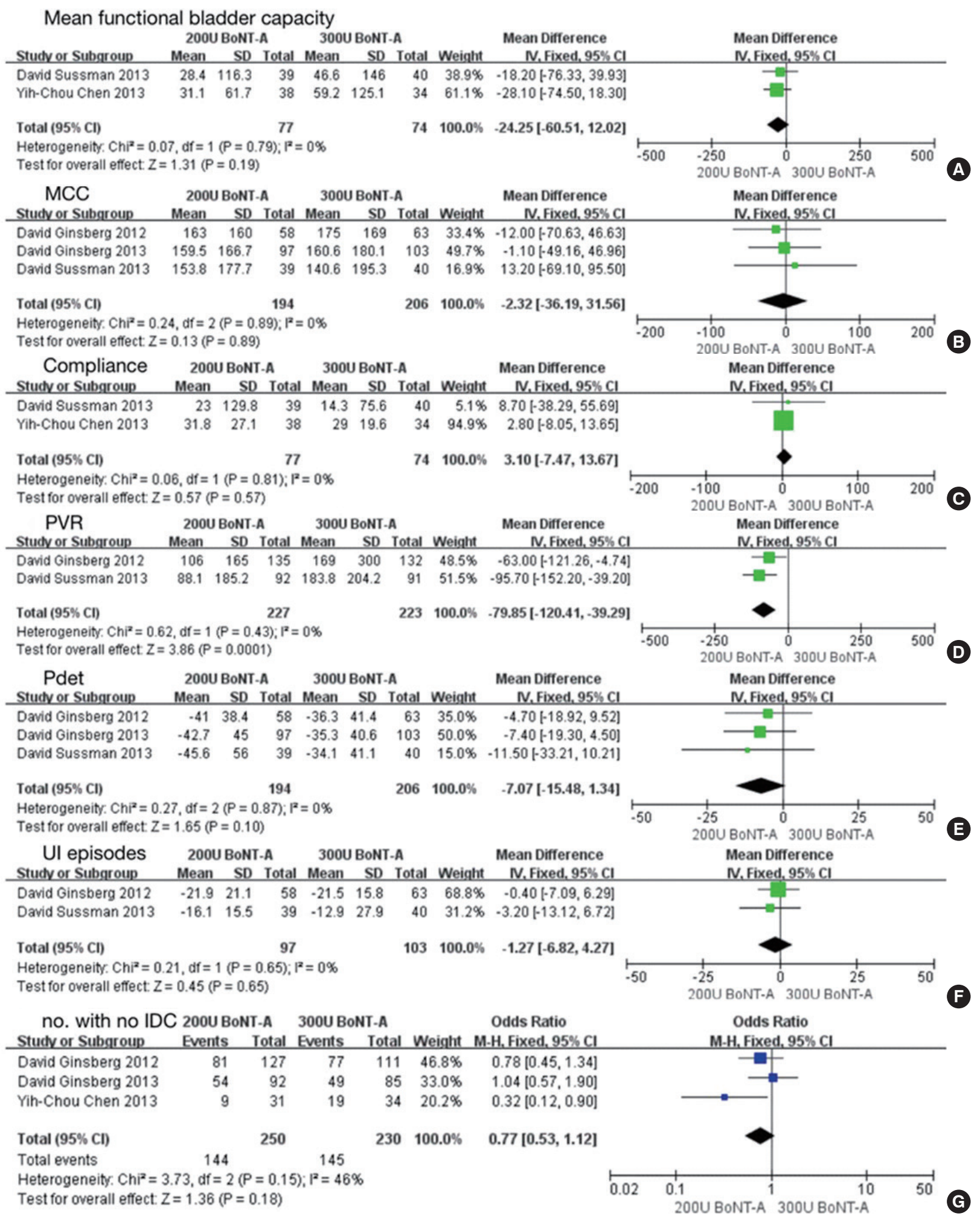

Fig. 6. Forest plots comparing the difference between $200 \mathrm{U}$ and $300 \mathrm{U}$ of onabotulinumtoxinA (BoNT-A) on mean functional bladder capacity (A), maximum cystometric capacity (MCC) (B), compliance (C), postvoid residual volume (PVR) (D), detrusor pressure (Pdet) (E), urinary incontinence (UI) episodes (F), and number of patients with no involuntary detrusor contraction (IDC) (G). SD, standard deviation; CI, confidence interval; df, degrees of freedom. 


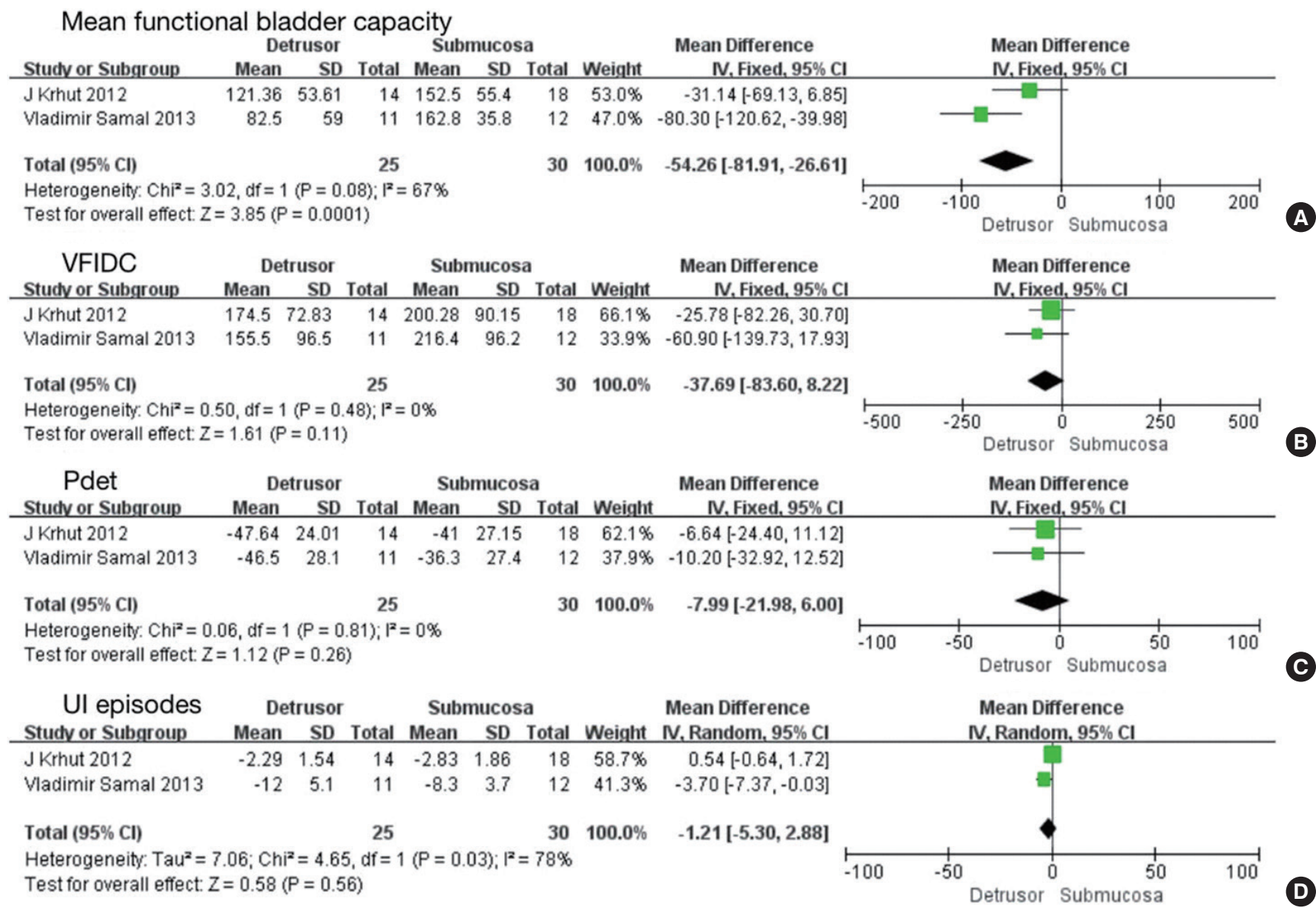

Fig. 7. Forest plots comparing the difference between injections into the detrusor versus submucosa on mean functional bladder capacity (A), volume at first involuntary detrusor contraction (VFIDC) (B), detrusor pressure (Pdet) (C), and urinary incontinence (UI) episodes (D). SD, standard deviation; CI, confidence interval; df, degrees of freedom.

0.00-0.17; $\mathrm{P}=0.0003)$, reduced $\mathrm{Qmax}(\mathrm{MD}, 3.34 ; 95 \% \mathrm{CI}$, 2.17-4.51; $\mathrm{P}<0.00001)$, and reduced incidence of DO (OR, 64.27; 95\% CI, 12.17-339.28; $\mathrm{P}<0.00001$ ) (Fig. 5).

Five other studies presented the effects of BoNT-A on I-QoL $[14,16,18,20,23]$. They were divided into 2 groups because they used different tools to evaluate I-QoL: a 22-item questionnaire and the International Prostatic Symptom Score questionnaire. Their results showed that BoNT-A was effective in improving IQoL, and this improvement was significant (Fig. 5F).

\section{BoNT-A dose: 200 U versus $300 \mathrm{U}$}

Four studies involving 919 participants included data comparing doses of $200 \mathrm{U}$ of BoNT-A versus $300 \mathrm{U}$ [9,12,14,18,25]. No significant difference was found between $200 \mathrm{U}$ and $300 \mathrm{U}$ of BoNT-A in mean functional bladder capacity (MD, 24.5; 95\% CI, -60.51 to $12.02 ; \mathrm{P}<0.19)$, MCC (MD, $-2.32 ; 95 \% \mathrm{CI},-36.19$ to 31.56; $\mathrm{P}<0.89$ ), compliance ( $\mathrm{MD}, 3.10 ; 95 \% \mathrm{CI},-7.47$ to 13.67; $\mathrm{P}<0.57$ ), PVR (MD, -79.85; 95\% CI, -120.41 to -39.29 ; $\mathrm{P}=0.0001$ ), Pdet (MD, $-7.07 ; 95 \% \mathrm{CI},-15.48$ to $1.34 ; \mathrm{P}<0.10)$, UI episodes (MD, $-1.27 ; 95 \% \mathrm{CI},-6.82$ to $4.27 ; \mathrm{P}=0.65)$ and the number of patients with no IDC (MD, 0.77; 95\% CI, 0.53-1.12; $\mathrm{P}=0.18$ ) (Fig. 6).

\section{Injections into the detrusor versus submucosa}

Two studies involving 55 participants included data comparing injections into the detrusor versus submucosa [19,24]. No significant difference was found in mean functional bladder capacity (MD, $-54.26 ; 95 \% \mathrm{CI},-81.91$ to $-26.61 ; \mathrm{P}=0.0001$ ), VFIDC (MD, -37.69 ; 95\% CI, -83.60 to 8.22 ; $\mathrm{P}<0.00001$ ), Pdet (MD, -7.99; 95\% CI, -21.98 to $6.00 ; \mathrm{P}=0.26$ ) and UI episodes (MD, $-1.21 ; 95 \% \mathrm{CI},-5.30$ to $2.88 ; \mathrm{P}=0.56$ ) (Fig. 7 ). 
Table 2. Complications of BoNT-A and placebo

\begin{tabular}{lccc}
\hline Adverse event & BoNT-A & Placebo & P-value $^{\mathrm{a})}$ \\
\hline Symptomatic UTI & $432 / 861(50.17)$ & $160 / 425(37.65)$ & 0.00002 \\
Urinary retention & $150 / 732(20.49)$ & $15 / 409(3.67)$ & 0.00000 \\
Hematuria & $57 / 861(6.62)$ & $15 / 425(3.53)$ & 0.02334 \\
Diarrhea & $41 / 732(5.60)$ & $19 / 409(4.65)$ & 0.48799 \\
Nausea & $40 / 732(5.46)$ & $8 / 409(1.96)$ & 0.00464 \\
Constipation & $38 / 732(5.19)$ & $18 / 409(4.40)$ & 0.55348 \\
Pyrexia & $38 / 761(4.99)$ & $16 / 396(4.04)$ & 0.34073 \\
Fatigue & $35 / 704(4.97)$ & $13 / 380(3.42)$ & 0.23639 \\
Headache & $34 / 732(4.64)$ & $15 / 409(3.67)$ & 0.43487 \\
Autonomic dysreflexia & $29 / 776(3.74)$ & $2 / 380(0.53)$ & 0.00150 \\
\hline
\end{tabular}

Values are presented as number (\%).

BoNT-A, onabotulinumtoxinA; UTI, urinary tract infection.

${ }^{\text {a) }} \mathrm{P}$-values were the outcomes of the chi-square tests for independence of each adverse event between the 2 groups.

\section{Safety}

We recorded all types of AEs and their proportions [9-28]. The chi-square test was used to evaluate differences between pairs of groups. Table 2 lists the top 10 AEs, including symptomatic urinary tract infection, urinary retention, hematuria, diarrhea, nausea, constipation, pyrexia, fatigue, headache, and autonomic dysreflexia. Nearly half of the AEs were not considered to have been treatment-related. Others could recover spontaneously or with only symptomatic treatment, and no AEs were life-threatening.

\section{DISCUSSION}

The neurotoxin Botulinum toxin, first described by van Ermengem in 1897, was extracted from the bacterium Clostridium botulinum $[29,30]$. BoNT-A, as a subtype, received FDA approval for the clinical treatment of NDO in 2011 [31]. BoNT-A inhibits neurotransmitter release from efferent nerve and sensory nerve endings. After BoNT-A injections, the expression of bladder muscarinic receptors $\mathrm{M} 2$ and M3 is reduced in humans with NDO [32]. For the above reasons, BoNT-A can induce the inhibition of DO and cause Pdet to decrease. Recent studies have reported it to have significant benefits on urodynamic parameters and clinical indices [33].

According to this meta-analysis, compared with baseline, BoNT-A was effective in increasing MCC, VFIDC, the voiding volume, compliance, I-QoL, the number of patients with $\mathrm{CD}$ and no IDC, and in decreasing Pdet, UI episodes, Qmax, and the incidence of DO [10,15-24]. Meanwhile, this meta-analysis further indicated that compared with placebo, BoNT-A was effective in increasing MCC, VFIDC, and the number of patients with no IDC, and in decreasing Pdet and UI episodes during 18 months of follow-up [8-14]. However, PVR was found to increase. The reason for this is that as MCC and VFIDC increased, the residual volume after voiding also increased. Additionally, since the dose used in clinical therapy remained controversial, our review mainly focused on comparing the most frequent doses (200 U and $300 \mathrm{U}$ ) [31]. Both doses were comparably effective in terms of outcomes for mean functional bladder capacity, MCC, compliance, PVR, Pdet, UI episodes, and the number of patients with no IDC $[9,12,14,18,25]$. Likewise, no significant difference was found between injection into the detrusor versus the submucosa in terms of mean functional bladder capacity, VFIDC, Pdet, and UI episodes [19,24].

In addition to the above-presented results, 2 RCTs involving 127 participants compared injection locations (sparing the trigone and excluding the trigone). One study compared $160 \mathrm{U}$ of BoNT-A injected into the bladder wall combined with $40 \mathrm{U}$ into the trigone versus the injection of $200 \mathrm{U}$ into the bladder wall, avoiding the trigone [16]. Both methods increased the number of patients with $\mathrm{CD}(+13$ vs. $+5, \mathrm{P}=0.01)$, improved IQoL (26.01 \pm 11.56 vs. $18.75 \pm 15.18)$, decreased UI episodes $(-5.22 \pm 0.91$ vs. $-4.68 \pm 1.06, P=0.01)$, and Pdet $(-33.34 \pm 20.27$ vs. $-28.02 \pm 15.18, \mathrm{P}=0.04)$. However, sparing the trigone was considered preferable [11]. The other study compared $200 \mathrm{U}$ injected into the detrusor combined with $100 \mathrm{U}$ into the trigone versus $300 \mathrm{U}$ injected into the detrusor, avoiding the trigone [23]. Sparing the trigone was more obviously preferable in terms of increasing patients with $\mathrm{CD}(+12$ vs. $+6, \mathrm{P}<0.001)$ and decreasing UI episodes $(0.98 \pm 0.45$ vs. $2.38 \pm 0.79, \mathrm{P}<0.001)$. However, no significant differences were found in MCC (384.6 \pm 49.7 vs. $406.5 \pm 55.4, \mathrm{P}=0.22)$, $\mathrm{Pdet}(35.0 \pm 3.6$ vs. $36.9 \pm 5.1$, $\mathrm{P}=0.21)$, I-QoL (2.22 \pm 0.55 vs. $2.22 \pm 0.65, \mathrm{P}=0.44)$ [22]. Considering the diversity of doses in the 2 studies, further research is needed.

Nevertheless, there were some limitations of our analysis. We considered conducting subgroup analyses based on quality assessment, as shown in Table 1. However, there were not enough data in each subgroup for such an analysis. Therefore, considering the same standard of quality assessments for all the studies and in order to avoid repeating our analyses, we summarized the primary characteristics and quality assessment results of all the included studies in Table 1. Furthermore, it seemed to be 
better to evaluate the MD of differences instead of the MD of values when comparing the effect of BoNT-A on baseline versus posttreatment values. However, most of the raw data from the included studies only provided the values after treatment, instead of the difference between baseline and after treatment. Therefore, we only evaluated the MDs.

In addition, heterogeneity could be generated by the subjects chosen, the location and dose of each site, and the loss of some negative data. Heterogeneity can be reduced by applying conscientious and rigorous procedures and sensitivity analyses, but it cannot be eliminated. Meanwhile, due to differences in the follow-up periods, the long-term effects were somewhat difficult to evaluate. Furthermore, the data in the studies included in this meta-analysis were insufficient to determine which dose and injection site were better for patients, so more highquality prospective trials are still needed [8].

In conclusion, BoNT-A is effective and safe in treating NDO after SCI in the short term. There were no significant differences between $200 \mathrm{U}$ or $300 \mathrm{U}$ of BoNT-A and between injecting into the detrusor or submucosa. More high-quality and refined RCTs are needed.

\section{AUTHOR CONTRIBUTION STATEMENT}

- Full access to all the data in the study and takes responsibility for the integrity of the data and the accuracy of the data analysis: GPL, $X Y W, Y Z$

- Study concept and design: GPL, XYW, YZ

- Acquisition of data: GPL, XYW

- Analysis and interpretation of data: GPL, XYW

- Drafting of the manuscript: $G P L$

- Critical revision of the manuscript for important intellectual content: GPL, XYW, YZ

- Statistical analysis: GPL, XYW

- Study supervision: $Y Z$

\section{REFERENCES}

1. Leitner L, Walter M, Freund P, Mehnert U, Michels L, Kollias S, et al. Protocol for a prospective magnetic resonance imaging study on supraspinal lower urinary tract control in healthy subjects and spinal cord injury patients undergoing intradetrusor onabotulinumtoxinA injections for treating neurogenic detrusor overactivity. BMC Urol 2014;14:68.

2. Tetreault LA, Karadimas S, Wilson JR, Arnold PM, Kurpad S, Det- tori JR, et al. The natural history of degenerative cervical myelopathy and the rate of hospitalization following spinal cord injury: an updated systematic review. Global Spine J 2017;7(3 Suppl):28S-34S. 3. Hu HZ, Granger N, Jeffery ND. Pathophysiology, clinical importance, and management of neurogenic lower urinary tract dysfunction caused by suprasacral spinal cord injury. J Vet Intern Med 2016;30:1575-88.

4. Schöps TF, Schneider MP, Steffen F, Ineichen BV, Mehnert U, Kessler TM. Neurogenic lower urinary tract dysfunction (NLUTD) in patients with spinal cord injury: long-term urodynamic findings. BJU Int 2015;115 Suppl 6:33-8.

5. Andy UU, Harvie HS, Smith AL, Propert KJ, Bogner HR, Arya LA. Validation of a self-administered instrument to measure adherence to anticholinergic drugs in women with overactive bladder. Neurourol Urodyn 2015;34:424-8.

6. Fergany LA, Shaker H, Arafa M, Elbadry MS. Does sacral pulsed electromagnetic field therapy have a better effect than transcutaneous electrical nerve stimulation in patients with neurogenic overactive bladder? Arab J Urol 2017;15:148-52.

7. Linsenmeyer TA. Use of botulinum toxin in individuals with neurogenic detrusor overactivity: state of the art review. J Spinal Cord Med 2013;36:402-19.

8. Yan HL, Zong HT, Cui YS, Li N, Zhang Y. Calcineurin inhibitor avoidance and withdrawal for kidney transplantation: a systematic review and meta-analysis of randomized controlled trials. Transplant Proc 2014;46:1302-13.

9. Ginsberg D, Cruz F, Herschorn S, Gousse A, Keppenne V, Aliotta P, et al. OnabotulinumtoxinA is effective in patients with urinary incontinence due to neurogenic detrusor overactivity [corrected] regardless of concomitant anticholinergic use or neurologic etiology. Adv Ther 2013;30:819-33.

10. Jia C, Liao LM, Chen G, Sui Y. Detrusor botulinum toxin A injection significantly decreased urinary tract infection in patients with traumatic spinal cord injury. Spinal Cord 2013;51:487-90.

11. Apostolidis A, Thompson C, Yan X, Mourad S. An exploratory, placebo-controlled, dose-response study of the efficacy and safety of onabotulinumtoxinA in spinal cord injury patients with urinary incontinence due to neurogenic detrusor overactivity. World J Urol 2013;31:1469-74.

12. Sussman D, Patel V, Del Popolo G, Lam W, Globe D, Pommerville P. Treatment satisfaction and improvement in health-related quality of life with onabotulinumtoxinA in patients with urinary incontinence due to neurogenic detrusor overactivity. Neurourol Urodyn 2013;32:242-9.

13. Cruz F, Herschorn S, Aliotta P, Brin M, Thompson C, Lam W, et al. 
Efficacy and safety of onabotulinumtoxinA in patients with urinary incontinence due to neurogenic detrusor overactivity: a randomised, double-blind, placebo-controlled trial. Eur Urol 2011;60:742-50.

14. Ginsberg D, Gousse A, Keppenne V, Sievert KD, Thompson C, Lam W, et al. Phase 3 efficacy and tolerability study of onabotulinumtoxinA for urinary incontinence from neurogenic detrusor overactivity. J Urol 2012;187:2131-9.

15. Chen SF, Chang CH, Kuo HC. Clinical efficacy and changes of urothelial dysfunction after repeated detrusor botulinum toxin A injections in chronic spinal cord-injured bladder. Toxins (Basel) 2016 May 30;8(6). pii: E164. https://doi.org/10.3390/toxins8060164.

16. Hui C, Keji X, Chonghe J, Ping T, Rubiao O, Jianweng Z, et al. Combined detrusortrigone BTX-A injections for urinary incontinence secondary to neurogenic detrusor overactivity. Spinal Cord 2016;54:46-50.

17. Sengoku A, Okamura K, Kimoto Y, Ogawa T, Namima T, Yamanishi $\mathrm{T}$, et al. Botulinum toxin $\mathrm{A}$ injection for the treatment of neurogenic detrusor overactivity secondary to spinal cord injury: multiinstitutional experience in Japan. Int J Urol 2015;22:306-9.

18. Chen YC, Kuo HC. The therapeutic effects of repeated detrusor injections between 200 or 300 units of onabotulinumtoxinA in chronic spinal cord injured patients. Neurourol Urodyn 2014;33:129-34.

19. Krhut J, Samal V, Nemec D, Zvara P. Intradetrusor versus suburothelial onabotulinumtoxinA injections for neurogenic detrusor overactivity: a pilot study. Spinal Cord 2012;50:904-7.

20. Chen CY, Liao CH, Kuo HC. Therapeutic effects of detrusor botulinum toxin A injection on neurogenic detrusor overactivity in patients with different levels of spinal cord injury and types of detrusor sphincter dyssynergia. Spinal Cord 2011;49:659-64.

21. Gamé X, Castel-Lacanal E, Bentaleb Y, Thiry-Escudié I, De Boissezon X, Malavaud B, et al. Botulinum toxin A detrusor injections in patients with neurogenic detrusor overactivity significantly decrease the incidence of symptomatic urinary tract infections. Eur Urol 2008;53:613-8.

22. Klaphajone J, Kitisomprayoonkul W, Sriplakit S. Botulinum toxin type A injections for treating neurogenic detrusor overactivity combined with low-compliance bladder in patients with spinal cord lesions. Arch Phys Med Rehabil 2005;86:2114-8.

23. Abdel-Meguid TA. Botulinum toxin-A injections into neurogenic overactive bladder--to include or exclude the trigone? A prospective, randomized, controlled trial. J Urol 2010;184:2423-8.

24. Šámal V, Mečl J, Šrám J. Submucosal administration of onabotulinumtoxinA in the treatment of neurogenic detrusor overactivity: pilot single-centre experience and comparison with standard injection into the detrusor. Urol Int 2013;91:423-8.

25. Herschorn S, Gajewski J, Ethans K, Corcos J, Carlson K, Bailly G, et al. Efficacy of botulinum toxin A injection for neurogenic detrusor overactivity and urinary incontinence: a randomized, double-blind trial. J Urol 2011;185:2229-35.

26. Schurch B, Denys P, Kozma CM, Reese PR, Slaton T, Barron RL. Botulinum toxin A improves the quality of life of patients with neurogenic urinary incontinence. Eur Urol 2007;52:850-8.

27. Schurch B, Denys P, Kozma CM, Reese PR, Slaton T, Barron R. Reliability and validity of the Incontinence Quality of Life questionnaire in patients with neurogenic urinary incontinence. Arch Phys Med Rehabil 2007;88:646-52.

28. Schurch B, de Sèze M, Denys P, Chartier-Kastler E, Haab F, Everaert K, et al. Botulinum toxin type a is a safe and effective treatment for neurogenic urinary incontinence: results of a single treatment, randomized, placebo controlled 6-month study. J Urol 2005;174:196-200.

29. Zakin E, Simpson D. Evidence on botulinum toxin in selected disorders. Toxicon 2018;147:134-40.

30. Smith CP, Boone TB, de Groat WC, Chancellor MB, Somogyi GT. Effect of stimulation intensity and botulinum toxin isoform on rat bladder strip contractions. Brain Res Bull 2003;61:165-71.

31. Nuanthaisong U, Abraham N, Goldman HB. Incidence of adverse events after high doses of onabotulinumtoxinA for multiple indications. Urology 2014;84:1044-8.

32. Khera M, Somogyi GT, Kiss S, Boone TB, Smith CP. Botulinum toxin A inhibits ATP release from bladder urothelium after chronic spinal cord injury. Neurochem Int 2004;45:987-93.

33. Do TP, Hvedstrup J, Schytz HW. Botulinum toxin: A review of the mode of action in migraine. Acta Neurol Scand 2018;137:442-51. 